\title{
Characterization of Fashion Themes Using Fuzzy Techniques for Designing New Human Centered Products
}

\author{
Y. Zhu ${ }^{1,2,3, *}$, D. Ruan ${ }^{4,5}$, X. Zeng ${ }^{1,2}$, L. Koehl ${ }^{1,2}$, C. Chaigneau ${ }^{3}$ \\ ${ }^{1}$ Univ Lille Nord de France, F-59000 Lille, France \\ ${ }^{2}$ Laboratoire Génie et Matériaux Textile (GEMTEX) \\ Ecole Nationale Supérieure des Arts et Industries Textiles (ENSAIT), F-59100 Roubaix, France \\ ${ }^{3}$ Damart Serviposte, F-59100 Roubaix, France \\ ${ }^{4}$ Belgian Nuclear Research Centre (SCK・CEN), \\ Boeretang 200, $2400 \mathrm{Mol}$, Belgium \\ ${ }^{5}$ Department of Applied Mathematics and Computer Science \\ Ghent University, 9000 Gent, Belgium
}

Received: 18-01-2010; Accepted: 25-05-2010

\begin{abstract}
Fabric selection plays an important role in fashion garment design. Designers often use both physical and normalized linguistic criteria for fabric selection. Perception and preference of consumers in their specific sociocultural context, expressed by fashion themes or emotional linguistic criteria, affect greatly new fashion product design. Modeling the relationship between linguistic design criteria and fashion themes of a brand image perceived by consumers becomes thus significant. For setting up this model, we first use fuzzy relations and correlation techniques to select the most relevant linguistic design criteria of fabric hand for each specific fashion theme. The selected criteria can then effectively reduce the complexity of the model and interpret consumer perception of fabrics. Finally, we use a weighted aggregation operator to predict the similarity degree between any new product and fashion themes. Compared with other models, the proposed method is more robust and easier to be interpreted with real data collected for design of senior T-shirt fabrics.
\end{abstract}

Keywords: Fabric selection, fashion garment design, data fusion, fuzzy sets, and similarity degree.

\section{Introduction}

Traditionally, designers select an appropriate fabric material based on their knowledge and experience for fashion product development. Automation of the fabric selection becomes an interesting research area for garment industry. In a garment company, key features on garment products can generally be characterized using three information sources: 1) physical measures by appropriate devices (numerical data), 2) normalized basic sensory evaluation data (normalized and neutral linguistic data), and 3) consumer perception on garment products (emotional linguistic data). The understanding of the relations between these information sources permits to identify consumer's behaviours on fashion themes and integrate them into the development of new garment products.

In a general context, the relationship between a textile product design and a consumer perception on garment products can be described in Fig. 1. The textile design criteria include physical properties such as shear stiffness, bending rigidity, thickness, and sensory aspects such as fabric hand evaluation, and visual evaluation. The physical properties are characterized by instrumental measures while the sensory linguistic criteria are determined by classical descriptive sensory evaluation methods $^{1,2}$ at different levels (fabric hand, vision, and olfaction).

*2, allée Louise et Victor Champier, BP 30329, 59056 Roubaix Cedex1, France. Tel: 33-320258689.E-mail: yijun.zhu@ensait.fr 


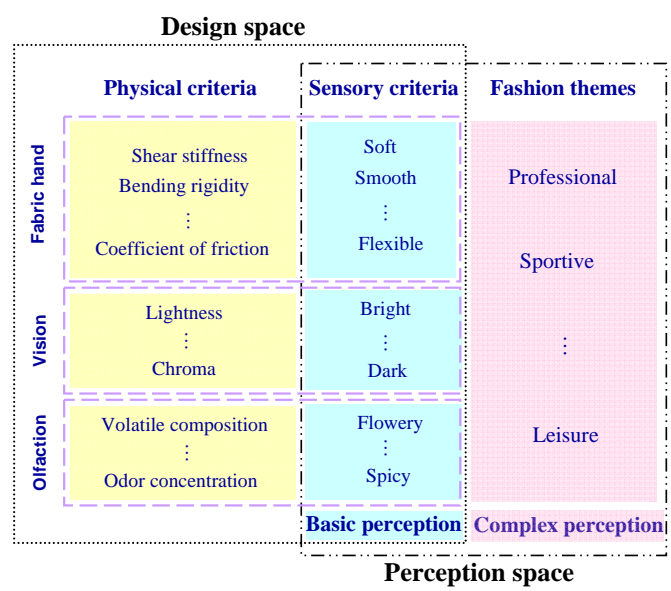

Fig. 1: Fig. 1. Relation between design space and perception space

However, the perception of consumers on garments products is not limited to the previous sensory linguistic criteria. It considers two levels:

(i) Basic perception on products, corresponding to normalized sensory criteria of design. It is independent of the social and cultural context of products and consumers.

(ii) Consumer perception in a social and cultural context, ${ }^{3}$ including a number of complex concepts such as comfort and well-being. In a specific context, each complex concept is defined by the marketing department according to its brand image, and it can be further decomposed into a set of ambiances or relatively concrete indicators. For example, "well-being" in garments is decomposed into "professional”, "sportive”, "protection", and "leisure".

Much research has been done for modelling the relationship between physical criteria and sensory descriptors to predict tactile basic perception according to mechanical properties. ${ }^{4,5,6,7,8}$ However, consumer perception in a specific socio-cultural context such as fashion themes is not taken into account in these methods. In practice, fabrics are generally selected by garment designers according to a number of physical and basic sensory criteria such as fabric hand and fabric appearance. The results of selected fabrics do not necessarily correspond to the desired fashion themes or the required brand image of garment products.

Hence, the characterization of the relationship between basic sensory criteria and fashion themes is particularly significant for the fashion garment design. The relevancy of sensory criteria to a given fashion theme is in fact usually considered as one of the most important elements for improving the brand image of garment products. In a new garment design, the conformity of perception between garments and their brand image enhances consumers' loyalty on the brand and reduces the risk and cost of design for the company.

In the existing literature, modelling of a complex relationship or system is often performed using artificial intelligent $^{9,10,11}$ from a set of representative learning data. However, in practice, these methods are not efficient in most of industrial applications, in which the quantity of learning data is often limited due to the restrictions of experimental cost and time. In this paper, we propose a new method to model the relationship between basic sensory criteria and fashion themes using fuzzy modeling techniques ${ }^{12,13,14,15}$ and aggregation techniques. ${ }^{16,17}$ It permits designers to find the most relevant basic sensory criteria at different levels (fabric hand, style, and color, etc.) for a given fashion theme in order to produce the most appropriate prototype meeting specific requirements of consumers.

We focus on the relationship between fabric hand criteria and fashion themes. First, sensory evaluation is organized to obtain similarity degrees given by a number of consumers between each fashion theme and prototypes. Then, these similarity degrees are aggregated as fuzzy sets, characterizing the evaluation score of all consumers. A round composition of fuzzy relation is applied to calculate the similarity between sensory criteria and fashion themes. A ranking list of these criteria for each theme is obtained. Then, an overall correlation coefficient and a procedure are proposed to remove the irrelevant sensory criteria. Finally, the predicted similarity degree between sensory criteria and fashion themes is modelled by a weighted aggregation operator. This model can effectively help designers to predict the fashion themes of garment products from specific fabric samples and select the most appropriate fabrics according to consumer requirements on fashion themes.

\section{A Fashion Product Design using Fuzzy Similarity Relations and Correlation Techniques}

\subsection{Formalization}

The relationship between basic sensory attributes and a specific fashion theme can be considered as a complex system in which the sensory attributes and the fashion theme are taken as input and output variables 
respectively (Fig. 2). The designers wish to identify this relationship in a systematic way in order to select fabric samples according to the values of the sensory attributes corresponding to the desired fashion theme.

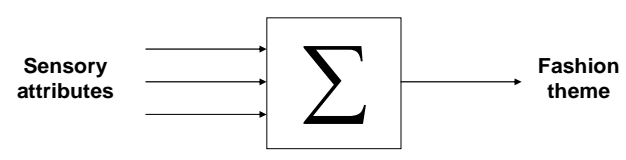

Fig. 2. The relationship between sensory attributes and one fashion theme

A brand image of the company is decomposed into a set of fashion themes describing products of a given collection. We have $T=\left\{t_{1}, t_{2}, \ldots, t_{b}\right\}$. For example, the brand image of a T-shirt collection is described by \{sportive, professional, leisure\}. In general, the brand image, the fashion themes and their associated fashion images are given by the marketing department of the company.

Apart from the space of fashion themes, industrial products can also be characterized by that of basic sensory criteria. The related concepts are defined as follows.

Let $S$ be a set of $n$ representative products of one collection, denoted as $S=\left\{s_{1}, \ldots, s_{n}\right\}$.

Let $A=\left\{a_{1}, \ldots, a_{p}\right\}$ be a set of $p$ basic sensory criteria or attributes describing the previous collection of products. For example, a fabric product is described by \{soft, flexible, smooth\}.

Let $R\left(s_{i}, a_{j}\right)=\left(e_{i j}\right)_{n \times p}$ be a matrix of evaluation scores for all the products of $S$ on the sensory descriptors of $A$, where $e_{i j}$ is the averaged evaluation score for the product $s_{i}$ on the descriptor $a_{j}$ given by a group of panelists. Each $e_{i j}$ varies between 0 and 1.1 means that $s_{i}$ is completely relevant to $a_{j}$. 0 means that $s_{i}$ is completely irrelevant to $a_{j}$. We consider that the difference between these trained panelists (experts) is very small because they have similar professional knowledge on the evaluated products.

Let $C=\left\{c_{1}, c_{2}, \ldots, c_{m}\right\}$ be a set of $m$ untrained panelists (or consumers) evaluating similarities between fabric samples and themes.

The corresponding evaluation values given by each consumer $c_{j}$ are denoted as $\left\{\tilde{x}_{i j k} \mid i=1, \ldots, n, k=1, \ldots, b\right\}$, where $\tilde{x}_{i j k}$ represents the evaluation score of the product $s_{i}$ related to the fashion theme $t_{k}$ given by the consumer $c_{j}$. It takes values from $\{C D$ : completely different, LC: a little close, C: close, VC: very close I: identical\}.

For all consumers, we obtain a distribution of their statistical evaluation results for all the linguistic values. In this case, the relationship between the fashion theme $t_{k}$ and the fabric samples $s_{i}$ is expressed by a fuzzy set distributed on the set $\left\{F_{1}=C D, F_{2}=L C, F_{3}=C, F_{4}=V C\right.$, $F_{5}=I$, i.e., $\tilde{X}_{i k}=\left(\frac{N\left(F_{1}\right)}{m} \frac{N\left(F_{2}\right)}{m} \ldots \frac{N\left(F_{5}\right)}{m}\right)$, where $N\left(F_{q}\right)$ is the number of evaluators selecting $F_{q}$ during the evaluation. Evidently, the sum of all components in $\tilde{X}_{i k}$ is 1 . For simplicity, we transform the linguistic values $F_{1}, \ldots, F_{5}$ into numerical values $\operatorname{Num}\left(F_{q}\right)$ uniformly distributed in $[0,1]$, i.e. $\operatorname{Num}\left(F_{1}\right)=0$, $\operatorname{Num}\left(F_{2}\right)=0.25, \quad \operatorname{Num}\left(F_{3}\right)=0.5, \quad \operatorname{Num}\left(F_{4}\right)=0.75$, $\operatorname{Num}\left(F_{5}\right)=1$. This definition conforms to the formalization of fuzzy variables in many real applications. Other choices for numerical values of $F_{q}$ 's can also be acceptable but the general trend of the final results should be similar. In this situation, two examples of the similarity between one fashion theme and one fabric sample are illustrated in Fig. 3. The peaks are the local maximums in the distribution.

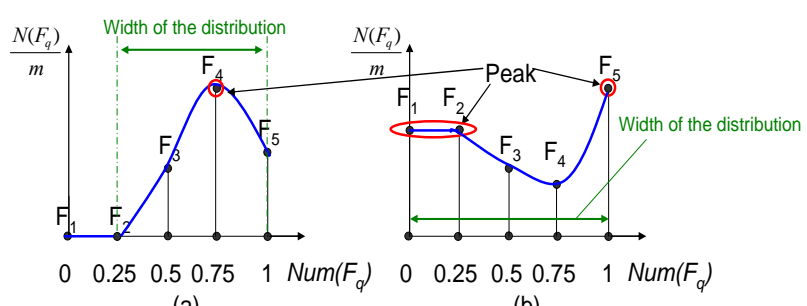

(a)

(b)

Fig. 3. Two fuzzy sets describing the relationship between $t_{k}$ and $s_{i}$

The performance of $\tilde{X}_{i k}$ is characterized by the corresponding distribution. The following principles should be taken into account. 1) If there exist more than one peak in the distribution of $\tilde{X}_{i k}$, then the evaluation results of different consumers are diversified and the relationship between the fashion theme $t_{k}$ and the fabric samples $s_{i}$ cannot be clearly identified by consumers. Otherwise, all evaluation data are centered on one peak and the relationship between $t_{k}$ and $s_{i}$ can be easily understood. 2) If the width of the distribution of $\tilde{X}_{i k}$ is small, then the evaluation results for all consumers are more concentrated and there is less ambiguity. Otherwise, the ambiguity is more important. In fact, 
these two principles are similar each other and the second is the refinement of the first. There is one peak in Fig. 3(a) and two peaks in Fig. 3(b). The width of the distribution of Fig. 3(a) is smaller than that of Fig. 3(b). So the evaluation results of Fig. 3(a) are more concentrated and more reliable than that of Fig. 3(b).

According to the above principles, we formally define the performance criteria of the fabric sample $s_{i}$ related to the fashion theme $t_{k}$ by

$$
V_{1 i k}=N_{-} \operatorname{peak}\left(\tilde{X}_{i k}\right) \text { and } V_{2 i k}=\operatorname{Var}\left(\tilde{X}_{i k}\right)
$$

In the previous definition, $N$ peak $\left(\tilde{X}_{i k}\right)$ and $\operatorname{Var}\left(\tilde{X}_{i k}\right)$ denote the number of peaks and the variance of the distribution in $\tilde{X}_{i k}$ respectively.

These performance criteria are important in the following computation because they characterize the quality of evaluation data relating the fabric sample $s_{i}$ to the fashion theme $t_{k}$. The performance of the fashion theme $t_{k}$ can be evaluated by aggregating the performance values corresponding to all the sensory descriptors, i.e.,

$$
V_{1 k}=\frac{1}{n} \sum_{i=1}^{n} V_{1 i k} \text { and } V_{2 k}=\frac{1}{n} \sum_{i=1}^{n} V_{2 i k}
$$

Low values of $V_{1 k}$ and $V_{2 k}$ mean that the relationship between the fashion theme $t_{k}$ and fabric sample $s_{i}$ is more understood by consumers and the definition of $t_{k}$ is more relevant to consumers. Otherwise, the fashion theme $t_{k}$ is less relevant for understanding of consumers.

For example, 10 consumers evaluate the relationship between two samples $s_{1}$ and $s_{2}$ and two fashion theme $t_{l}$ "warmth" and $t_{2}$ "leisure". And consumers take values from $\{C D, L C, C, V C, I\}$. The corresponding fuzzy values are given in Table 1.

Table 1. Distribution of fuzzy similarities of products with fashion themes

\begin{tabular}{|c|c|c|c|c|c|}
\hline & $F_{1}$ & $F_{2}$ & $F_{3}$ & $F_{4}$ & $F_{5}$ \\
\hline$\tilde{X}_{11}$ & 0 & 0 & 0.1 & 0.2 & 0.7 \\
\hline$\tilde{X}_{21}$ & 0.2 & 0.6 & 0.1 & 0.1 & 0 \\
\hline$\tilde{X}_{12}$ & 0.3 & 0.2 & 0.2 & 0.1 & 0.2 \\
\hline$\tilde{X}_{22}$ & 0.3 & 0.2 & 0.1 & 0.3 & 0.1 \\
\hline
\end{tabular}

From the above results, the evaluation scores between the fashion theme $t_{1}$ and the sample $s_{1}$ are concentrated around $F_{5}$ and those of $t_{1}$ and $s_{2}$ are concentrated around $F_{2}$. We consider that the relationship between $t_{1}$ and these two samples is clearly defined because there only exists one peak in the distribution of $\tilde{X}_{11}$ and $\tilde{X}_{21}$. Moreover, the relationship between $t_{2}$ and these two samples is not clearly identified by consumers because the corresponding distributions include two peaks.

Table 2. Performance value of fashion themes

\begin{tabular}{|c|c|c|}
\hline & $t_{1}$ & $t_{2}$ \\
\hline$V_{1 k}$ & 1 & 2 \\
\hline$V_{2 k}$ & 0.05 & 0.1 \\
\hline
\end{tabular}

Next, the values of $V_{1 k}$ and $V_{2 k}$ are calculated and shown in Table 2 . The relationship between the fashion theme $t_{l}$ and samples is easier to be identified than that of $t_{2}$ by consumers because the distribution of consumers' results is concentrated on one peak $\left(V_{1 k}=1\right)$ and small variance of the distribution result (low value of $\left.V_{2 k}\right)$.

\subsection{Similarity between sensory descriptors and fashion themes using fuzzy relations}

The deffuzzified similarity between product $s_{i}$ and a specific fashion theme $t_{k}$ can be obtained by

$$
\mathrm{R}\left(\mathrm{s}_{\mathrm{i}}, \mathrm{t}_{\mathrm{k}}\right)=\sum_{q=1}^{5} \frac{N\left(F_{q}\right)}{m} * \operatorname{Num}\left(F_{q}\right)
$$

In fact, it is the average of the numerical similarity degrees given by all the evaluators. As far we have the relation between descriptors and products and that between products and fashion themes. According to the fuzzy relation theory, ${ }^{18}$ the similarity between a sensory descriptor $a_{j}$ and the fashion theme $t_{k}$ can be defined by

$$
R\left(a_{j}, t_{k}\right)=\underset{i=1}{\vee p}\left(R\left(s_{i}, a_{j}\right) \wedge R\left(s_{i}, t_{k}\right)\right)
$$

The value of $R\left(a_{j}, t_{k}\right)$ varies between 0 and 1 . The closer $R\left(a_{j}, t_{k}\right)$ is to 1 , the more the sensory descriptor $a_{j}$ is relevant to the fashion theme $t_{k}$. Otherwise, the closer $R\left(a_{j}, t_{k}\right)$ is to 0 , the more $a_{j}$ is irrelevant to $t_{k}$.

For example, we only have two samples $s_{1}, s_{2}$, two sensory descriptors $a_{1}, a_{2}$, and two fashion themes $t_{1}, t_{2}$. The relations $R\left(s_{i}, a_{j}\right)$ and $R\left(s_{i}, t_{k}\right)$ (the similarity values) have been obtained from the corresponding evaluations and the results are shown in Table 3. 
Table 3. Relation of $R\left(s_{i}, a_{j}\right)$ and $R\left(s_{i}, t_{k}\right)$

\begin{tabular}{|c|c|c|c|c|c|}
\hline$R\left(a_{j}, s_{i}\right)$ & $s_{1}$ & $s_{2}$ & $R\left(s_{i}, t_{k}\right)$ & $t_{1}$ & $t_{2}$ \\
\hline$a_{l}$ & 0.3 & 0.7 & $s_{I}$ & 0.1 & 0.7 \\
\hline$a_{2}$ & 0.3 & 0.8 & $s_{2}$ & 0.9 & 0.3 \\
\hline$R\left(a_{j}, t_{k}\right)$ & $t_{1}$ & $t_{2}$ & & & \\
\hline$a_{1}$ & 0.7 & 0.3 & & & \\
\hline$a_{2}$ & 0.8 & 0.3 & & & \\
\hline
\end{tabular}

From Table 3, we can see that, although sample $s_{1}$ has a small similarity with sensory descriptor $a_{1}$ and fashion theme $t_{l}, R\left(a_{l}, t_{l}\right)$ is rather big because of the big similarities of sample $s_{2}\left(R\left(s_{2}, a_{2}\right)\right.$ and $\left.R\left(s_{2}, t_{1}\right)\right)$ in recompense. So the similarities between sensory descriptors and fashion themes are obtained by considering the whole set of samples and their similarity values with sensory descriptors and fashion themes.

\subsection{Removal of correlations between sensory descriptors}

For each pair of sensory descriptors (denoted as $a_{i}$ and $a_{j}$ ), we calculate their linear correlation coefficient, denoted as $c c_{i, j}$. These correlations between sensory descriptors are independent of the fashion themes. Thus, the overall correlation coefficient (OCC) of sensory descriptor $a_{j}$ is calculated by

$$
o c c_{j}=\max _{i \neq j}\left\{c c_{i, j}\right\}
$$

Then, we propose a procedure for removing sensory descriptors strongly correlated with others. The principle of this procedure is illustrated as follows.

We rank all the sensory descriptors according to their similarity values calculated using the method of Section 2.2.

If $R\left(a_{i}, t_{k}\right)>R\left(a_{j}, t_{k}\right)>\gamma$ and $c c_{i, j}=o c c_{j}>\delta$, then the descriptor $a_{j}$ is relevant to $t_{k}$ and strongly correlated with $a_{i}$, and it should be removed from the ranking list.

If $R\left(a_{i}, t_{k}\right)<\gamma$, then the descriptor $a_{j}$ is irrelevant to $t_{k}$, and should be removed from the ranking list.

$\gamma$ denotes the threshold of the similarity degree between a sensory descriptor and a fashion theme while $\delta$ that of the correlation coefficient between two sensory descriptors. They are defined by experts according to the real situation. If they wish to obtain fewer relevant descriptors, they should give big values of $\gamma$ (strong restriction). If they hope that the extracted relevant descriptors are more independent and less correlated between them, they need to give big values of $\delta$. According to this principle, the ranked descriptors obtained in the final list are the independent variables relevant to the fashion theme.

\subsection{Modelling the relationship between sensory descriptors and fashion themes}

According to the rules given in Section 2.3, we obtain $g$ sensory descriptors $a_{1}, \ldots, a_{g}$ relevant to $t_{k}$. We suppose that these descriptors are already ranked by their similarity values with $t_{k}$ in a descending order. Next, we wish to determine the corresponding weights denoted as $U=\left(u_{1}, \ldots u_{g}\right)$ for exploiting better the importance of each descriptor and aggregating these descriptors more reasonably. The weights are defined according to the following principle: 1 ) If the similarity of the descriptor $a_{i}$ and the theme $t_{k}$ is big or if the rank of this similarity value is high, then the corresponding weight is big. Otherwise, this weight is small. 2) If two neighbouring similarity values in the ranking list are identical, their weights should be the same. Having denoted the ranking order as $O=\left(o_{1}, o_{2}, \ldots, o_{g}\right)=(g, g-1, \ldots, 1\}$, we calculate the corresponding weights as follows

$$
u_{i}=\left\{\begin{array}{cc}
o_{i} & R\left(a_{i}, t_{k}\right) \neq R\left(a_{i+1}, t_{k}\right) \\
\frac{o_{i}+o_{i+1}}{2} & R\left(a_{i}, t_{k}\right)=R\left(a_{i+1}, t_{k}\right)
\end{array}\right.
$$

For example, we have the similarity values of four descriptors with $t_{1}: 0.8,0.7,0.7$, and 0.6 . The corresponding ranking order $O=\{4,3,2,1\}$. Using eq.(6), we obtain the corresponding weight $4,2.5,2.5$, 1. The difference of specific similarity values related to different descriptors is not significant. In this situation, the previous way of weight definition is just to strengthen the importance of the ranking order so that each descriptor can be better differentiated from another.

Next, we normalize the weight $u_{i}$ as follows.

$$
\tilde{u}_{i}=\frac{u_{i}}{\sum_{i=1}^{g} u_{i}}
$$

After the normalization, we have $\sum_{i=1}^{g} \tilde{u}_{i}=1$.

We consider that the similarity between any product and a specific fashion theme can be expressed by the weighted average of the similarity values between this product and all the relevant sensory descriptors. In this situation, we have

$$
R^{\prime}\left(s_{j}, t_{k}\right)=\sum_{i=1}^{g} \tilde{u}_{i} R\left(s_{j}, a_{i}\right)
$$


where $R^{\prime}\left(s, t_{k}\right)$ is the predicted similarity value between $s$ and $t_{k}$.

For a new product, we have

$$
R^{\prime}\left(s, t_{k}\right)=\sum_{i=1}^{g} \tilde{u}_{i} R\left(s, a_{i}\right)
$$

where $s$ represents the new product and $R\left(s, a_{i}\right)$ is obtained by the classic sensory evaluation method.

In this way, for any new product, we can easily estimate its similarity with a given fashion theme from the corresponding sensory evaluation results on the relevant descriptors.

In practice, the distribution of the predicted similarity degrees of $s_{i}$ 's with $t_{k}$, calculated from eq.(9), is similar with that of the real similarity degrees of the same products with $t_{k}$ evaluated by experts. In this situation, for any product $s$, the relation between $R$ ' $\left(s, t_{k}\right)$ and $R_{\text {pre }}\left(s, t_{k}\right)$ can be set up by transforming these two data distributions on the same scale. Therefore, we first normalize $R^{\prime}\left(s, t_{k}\right)$ into the scale of $[0,1]$ using $\mathrm{R}^{\prime}\left(\mathrm{s}, \mathrm{t}_{\mathrm{k}}\right)-\min \left(\mathrm{R}^{\prime}\left(\mathrm{s}_{\mathrm{j}}, t_{k}\right)\right)$

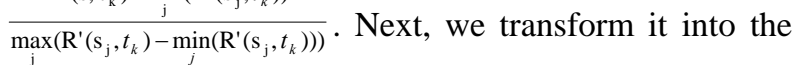
scale of the real similarity degrees by

$$
\begin{gathered}
\mathrm{R}_{\text {pre }}\left(\mathrm{s}, \mathrm{t}_{\mathrm{k}}\right)=\min _{j}\left(\mathrm{R}\left(\mathrm{s}_{\mathrm{j}}, t_{k}\right)\right)+\left(\max _{j}\left(\mathrm{R}\left(\mathrm{s}_{\mathrm{j}}, t_{k}\right)\right)-\min \left(\mathrm{R}\left(\mathrm{s}_{\mathrm{j}}, t_{k}\right)\right)\right) \\
* \frac{\mathrm{R}^{\prime}\left(\mathrm{s}, \mathrm{t}_{\mathrm{k}}\right)-\min _{\mathrm{j}}\left(\mathrm{R}^{\prime}\left(\mathrm{s}_{\mathrm{j}}, t_{k}\right)\right)}{\max _{\mathrm{j}}\left(\mathrm{R}^{\prime}\left(\mathrm{s}_{\mathrm{j}}, t_{k}\right)-\min _{j}\left(\mathrm{R}^{\prime}\left(\mathrm{s}_{\mathrm{j}}, t_{k}\right)\right)\right)} \\
\text { where } j \in[1, \ldots, n], \quad k \in[1, \ldots, b]
\end{gathered}
$$

In eq.(10), $R_{\text {pre }}\left(s, t_{k}\right)$ is the normalized similarity degree between $s$ and $t_{k}$. It can be considered as an estimation of the real similarity of the product $s$ with the fashion theme $t_{k}$.

Closer the value of $R_{\text {pre }}\left(s, t_{k}\right)$ is to 1 , more the new product $s$ is relevant to the fashion theme $t_{k}$. Otherwise, $s$ is irrelevant to $t_{k}$.

This modelling procedure can be summarized in five steps:

Step 1: Verify the performance of fashion themes using eq.(1) and eq.(2).

Step 2: Calculate the similarity degree between sensory descriptors and fashion themes using eq.(3) and eq.(4).

Step 3: Select the relevant and independent sensory descriptors related to a given fashion theme using the procedure of Section 2.3.

Step 4: Calculate the similarity degree between a new product and the fashion theme using a weighted aggregation operator eq.(6), eq.(7), eq.(8) and eq.(9).

Step 5: Normalize the similarity degree calculated in Step 4 using eq.(10).

\section{An Industrial Application}

The proposed method is applied to one real application of a company. We select six fashion themes describing the corresponding brand image and 11 fabrics for sensory evaluation on fabric hand. 13 sensory linguistic attributes are generated and used for evaluating these products. 20 consumers give similarity degrees between fabrics and fashion themes. The experts' evaluation results for 11 fabrics with 13 sensory attributes are

\begin{tabular}{|c|c|c|c|c|c|c|c|}
\hline$R\left(s_{j}, a_{i}\right)$ & $a_{1}$ & $a_{2}$ & $a_{3}$ & $a_{4}$ & $a_{5}$ & $a_{6}$ & $a_{7}$ \\
\hline$s_{1}$ & 0.35 & 0.39 & 0.41 & 0.34 & 0.47 & 0.22 & 0.94 \\
\hline$s_{2}$ & 0.03 & 0.04 & 0.16 & 0.81 & 0.19 & 0.17 & 0.55 \\
\hline$s_{3}$ & 0.92 & 0.63 & 0.72 & 0.12 & 0.90 & 0.47 & 0.49 \\
\hline$s_{4}$ & 0.47 & 0.64 & 0.63 & 0.25 & 0.61 & 0.41 & 0.62 \\
\hline$s_{5}$ & 0.13 & 0.13 & 0.27 & 0.50 & 0.44 & 0.16 & 0.83 \\
\hline$s_{6}$ & 0.99 & 0.52 & 0.63 & 0.15 & 0.91 & 0.31 & 0.73 \\
\hline$s_{7}$ & 0.15 & 0.62 & 0.73 & 0.24 & 0.37 & 0.68 & 0.37 \\
\hline$s_{8}$ & 0.70 & 0.12 & 0.15 & 0.92 & 0.84 & 0.20 & 0.24 \\
\hline$s_{9}$ & 0.00 & 0.99 & 0.98 & 0.00 & 0.09 & 0.82 & 0.09 \\
\hline$s_{10}$ & 0.73 & 0.67 & 0.70 & 0.16 & 0.76 & 0.32 & 0.76 \\
\hline$s_{11}$ & 0.51 & 0.59 & 0.54 & 0.38 & 0.53 & 0.26 & 0.76 \\
\hline$R\left(s_{j}, a_{i}\right)$ & $a_{8}$ & $a_{9}$ & $a_{10}$ & $a_{11}$ & $a_{12}$ & $a_{13}$ & \\
\hline$s_{1}$ & 0.63 & 0.67 & 0.70 & 0.37 & 0.25 & 0.62 & \\
\hline$s_{2}$ & 0.67 & 0.81 & 0.02 & 0.21 & 0.15 & 0.94 & \\
\hline$s_{3}$ & 0.26 & 0.30 & 0.64 & 0.91 & 0.86 & 0.08 & \\
\hline$s_{4}$ & 0.46 & 0.60 & 0.61 & 0.45 & 0.45 & 0.12 & \\
\hline$s_{5}$ & 0.69 & 0.72 & 0.26 & 0.40 & 0.34 & 0.63 & \\
\hline$s_{6}$ & 0.10 & 0.25 & 0.64 & 0.99 & 0.98 & 0.00 & \\
\hline$s_{7}$ & 0.57 & 0.71 & 0.42 & 0.35 & 0.35 & 0.23 & \\
\hline$s_{8}$ & 0.01 & 0.02 & 0.32 & 0.62 & 0.86 & 0.27 & \\
\hline$s_{9}$ & 0.99 & 0.99 & 0.17 & 0.10 & 0.02 & 0.82 & \\
\hline$s_{10}$ & 0.47 & 0.57 & 0.76 & 0.61 & 0.42 & 0.06 & \\
\hline$s_{11}$ & 0.55 & 0.68 & 0.72 & 0.44 & 0.30 & 0.25 & \\
\hline
\end{tabular}
given in Table 4, and the consumer's evaluation on similarity between the products and the six fashion themes are shown in Table 5.

Table 4 . Similarity value between $a_{j}$ and $s_{i}$ 
Table 5. Distribution of fuzzy similarities with six fashion themes for all the products

\begin{tabular}{|c|c|c|c|c|c|c|c|c|}
\hline & $\tilde{X}_{11}$ & $\tilde{X}_{21}$ & $\tilde{X}_{31}$ & $\tilde{X}_{41}$ & $\tilde{X}_{51}$ & $\tilde{X}_{61}$ & $\tilde{X}_{71}$ & $\tilde{X}_{81}$ \\
\hline$F_{1}$ & 0.05 & 0.75 & 0 & 0 & 0.4 & 0 & 0.2 & 0 \\
\hline$F_{2}$ & 0.25 & 0.2 & 0.05 & 0.05 & 0.4 & 0 & 0.35 & 0.1 \\
\hline$F_{3}$ & 0.3 & 0 & 0 & 0.15 & 0.15 & 0 & 0.3 & 0.35 \\
\hline$F_{4}$ & 0.3 & 0.05 & 0.1 & 0.6 & 0.05 & 0.05 & 0.15 & 0.3 \\
\hline$F_{5}$ & 0.1 & 0 & 0.85 & 0.2 & 0 & 0.95 & 0 & 0.25 \\
\hline & $\tilde{X}_{91}$ & $\tilde{X}_{101}$ & $\tilde{X}_{111}$ & $\tilde{X}_{12}$ & $\tilde{X}_{22}$ & $\tilde{X}_{32}$ & $\tilde{X}_{42}$ & $\tilde{X}_{52}$ \\
\hline$F_{1}$ & 0.55 & 0 & 0.05 & 0.05 & 0.1 & 0.3 & 0.05 & 0.05 \\
\hline$F_{2}$ & 0.25 & 0.05 & 0.1 & 0.1 & 0.35 & 0.15 & 0.1 & 0.1 \\
\hline$F_{3}$ & 0.15 & 0.25 & 0.35 & 0.5 & 0.3 & 0.35 & 0.55 & 0.25 \\
\hline$F_{4}$ & 0.05 & 0.35 & 0.3 & 0.25 & 0.2 & 0.15 & 0.2 & 0.4 \\
\hline$F_{5}$ & 0 & 0.35 & 0.2 & 0.1 & 0.05 & 0.05 & 0.1 & 0.2 \\
\hline & $\tilde{X}_{62}$ & $\tilde{X}_{72}$ & $\tilde{X}_{82}$ & $\tilde{X}_{92}$ & $\tilde{X}_{102}$ & $\tilde{X}_{112}$ & $\tilde{X}_{13}$ & $\tilde{X}_{23}$ \\
\hline$F_{1}$ & 0.3 & 0 & 0.65 & 0 & 0 & 0.1 & 0 & 0.3 \\
\hline$F_{2}$ & 0.5 & 0.1 & 0.2 & 0.25 & 0.2 & 0.1 & 0.2 & 0.25 \\
\hline$F_{3}$ & 0.1 & 0.15 & 0.1 & 0.2 & 0.3 & 0.35 & 0.25 & 0.05 \\
\hline$F_{4}$ & 0.1 & 0.35 & 0 & 0.3 & 0.5 & 0.35 & 0.45 & 0.35 \\
\hline$F_{5}$ & 0 & 0.4 & 0.05 & 0.25 & 0 & 0.1 & 0.1 & 0.05 \\
\hline & $\tilde{X}_{33}$ & $\tilde{X}_{43}$ & $\tilde{X}_{53}$ & $\tilde{X}_{63}$ & $\tilde{X}_{73}$ & $\tilde{X}_{83}$ & $\tilde{X}_{93}$ & $\tilde{X}_{103}$ \\
\hline$F_{1}$ & 0.05 & 0.05 & 0.15 & 0 & 0 & 0.6 & 0.15 & 0 \\
\hline$F_{2}$ & 0.15 & 0.15 & 0.25 & 0.15 & 0.3 & 0.25 & 0.25 & 0.15 \\
\hline$F_{3}$ & 0.15 & 0.25 & 0.1 & 0.35 & 0.2 & 0.1 & 0.1 & 0.25 \\
\hline$F_{4}$ & 0.3 & 0.4 & 0.4 & 0.35 & 0.35 & 0.05 & 0.25 & 0.4 \\
\hline$F_{5}$ & 0.35 & 0.15 & 0.1 & 0.15 & 0.15 & 0 & 0.25 & 0.2 \\
\hline & $\tilde{X}_{113}$ & $\tilde{X}_{14}$ & $\tilde{X}_{24}$ & $\tilde{X}_{34}$ & $\tilde{X}_{44}$ & $\tilde{X}_{54}$ & $\tilde{X}_{64}$ & $\tilde{X}_{74}$ \\
\hline$F_{1}$ & 0 & 0.2 & 0 & 0.65 & 0.25 & 0.1 & 0.75 & 0 \\
\hline$F_{2}$ & 0.05 & 0.2 & 0 & 0.25 & 0.45 & 0.1 & 0.15 & 0.1 \\
\hline$F_{3}$ & 0.3 & 0.2 & 0.15 & 0.05 & 0.2 & 0.05 & 0.05 & 0.15 \\
\hline$F_{4}$ & 0.5 & 0.2 & 0.3 & 0.05 & 0.1 & 0.45 & 0.05 & 0.35 \\
\hline$F_{5}$ & 0.15 & 0.2 & 0.55 & 0 & 0 & 0.3 & 0 & 0.4 \\
\hline & $\tilde{X}_{84}$ & $\tilde{X}_{94}$ & $\tilde{X}_{104}$ & $\tilde{X}_{114}$ & $\tilde{X}_{15}$ & $\tilde{X}_{25}$ & $\tilde{X}_{35}$ & $\tilde{X}_{45}$ \\
\hline$F_{1}$ & 0.75 & 0 & 0.25 & 0.15 & 0 & 0.45 & 0 & 0.05 \\
\hline$F_{2}$ & 0.2 & 0.1 & 0.45 & 0.35 & 0.2 & 0.55 & 0.05 & 0 \\
\hline$F_{3}$ & 0.05 & 0.05 & 0.25 & 0.15 & 0.3 & 0 & 0.05 & 0.3 \\
\hline$F_{4}$ & 0 & 0.4 & 0.05 & 0.25 & 0.5 & 0 & 0.3 & 0.55 \\
\hline$F_{5}$ & 0 & 0.45 & 0 & 0.1 & 0 & 0 & 0.6 & 0.1 \\
\hline
\end{tabular}

\begin{tabular}{|c|c|c|c|c|c|c|c|c|}
\hline & $\tilde{X}_{55}$ & $\tilde{X}_{65}$ & $\tilde{X}_{75}$ & $\tilde{X}_{85}$ & $\tilde{X}_{95}$ & $\tilde{X}_{105}$ & $\tilde{X}_{115}$ & $\tilde{X}_{16}$ \\
\hline$F_{1}$ & 0.2 & 0 & 0.1 & 0.05 & 0.3 & 0.05 & 0 & 0 \\
\hline$F_{2}$ & 0.4 & 0 & 0.2 & 0.2 & 0.4 & 0.05 & 0.15 & 0.15 \\
\hline$F_{3}$ & 0.25 & 0 & 0.3 & 0.15 & 0.2 & 0.15 & 0.25 & 0.2 \\
\hline$F_{4}$ & 0.15 & 0.3 & 0.4 & 0.3 & 0.1 & 0.7 & 0.6 & 0.45 \\
\hline \multirow[t]{2}{*}{$F_{5}$} & 0 & 0.7 & 0 & 0.3 & 0 & 0.05 & 0 & 0.2 \\
\hline & $\tilde{X}_{26}$ & $\tilde{X}_{36}$ & $\tilde{X}_{46}$ & $\tilde{X}_{56}$ & $\tilde{X}_{66}$ & $\tilde{X}_{76}$ & $\tilde{X}_{86}$ & $\tilde{X}_{96}$ \\
\hline$F_{1}$ & 0.1 & 0.05 & 0 & 0 & 0.1 & 0 & 0.6 & 0.05 \\
\hline$F_{2}$ & 0 & 0.3 & 0.15 & 0 & 0.35 & 0.05 & 0.2 & 0 \\
\hline$F_{3}$ & 0.25 & 0.35 & 0.3 & 0.1 & 0.5 & 0.25 & 0.15 & 0.1 \\
\hline$F_{4}$ & 0.4 & 0.3 & 0.4 & 0.6 & 0.05 & 0.35 & 0 & 0.45 \\
\hline \multirow[t]{2}{*}{$F_{5}$} & 0.25 & 0 & 0.15 & 0.3 & 0 & 0.35 & 0.05 & 0.4 \\
\hline & $\tilde{X}_{106}$ & $\tilde{X}_{116}$ & & & & & & \\
\hline$F_{1}$ & 0 & 0 & & & & & & \\
\hline$F_{2}$ & 0.2 & 0.15 & & & & & & \\
\hline$F_{3}$ & 0.45 & 0.25 & & & & & & \\
\hline$F_{4}$ & 0.35 & 0.55 & & & & & & \\
\hline$F_{5}$ & 0 & 0.05 & & & & & & \\
\hline
\end{tabular}

Next, we follow the steps of the previous modelling procedure for calculating different criteria.

Step 1: Performance verification of the fashion themes

First, we calculate the performance of the fashion themes using eq.(1) and eq.(2). We can see that for each theme there is only one peak in the distribution of the evaluation scores, and the variance of the distribution is small. All the fashion themes are well understood by the consumers.

Table 6. Performance criteria of fashion themes

\begin{tabular}{|c|c|c|c|c|c|c|}
\hline & $t_{1}$ & $t_{2}$ & $t_{3}$ & $t_{4}$ & $t_{5}$ & $t_{6}$ \\
\hline$V_{1 k}$ & 1 & 1 & 1 & 1 & 1 & 1 \\
\hline$V_{2 k}$ & 0.08 & 0.10 & 0.10 & 0.08 & 0.07 & 0.08 \\
\hline
\end{tabular}

First, we calculate the performance of the fashion themes using eq.(1) and eq.(2). We can see that for each theme there is only one peak in the distribution of the evaluation scores, and the variance of the distribution is small. All the fashion themes are well understood by the consumers. 
Table 7. Similarity between the descriptors and the fashion theme $t_{1}$ and the overall correlation coefficients between descriptors $(\delta=0.5, \gamma=0.5)$

\begin{tabular}{|c|c|}
\hline$a_{i}$ & $R\left(a_{i}, t_{l}\right)$ \\
\hline$a_{1}$ & 0.988 \\
\hline$a_{11}$ & 0.987 \\
\hline$a_{12}$ & 0.981 \\
\hline$a_{5}$ & 0.907 \\
\hline$a_{7}$ & 0.750 \\
\hline$a_{10}$ & 0.750 \\
\hline$a_{3}$ & 0.716 \\
\hline$a_{4}$ & 0.675 \\
\hline$a_{2}$ & 0.675 \\
\hline$a_{9}$ & 0.625 \\
\hline$a_{8}$ & 0.551 \\
\hline$a_{13}$ & 0.538 \\
\hline$a_{6}$ & 0.466 \\
\hline
\end{tabular}

\begin{tabular}{|c|c|}
\hline$c c_{i, j}$ & $o c c_{j k}$ \\
\hline$c c_{1,5}$ & 0.964 \\
\hline$c c_{2,3}$ & 0.978 \\
\hline$c c_{3,2}$ & 0.978 \\
\hline$c c_{4,3}$ & 0.936 \\
\hline$c c_{5,1}$ & 0.964 \\
\hline$c c_{6,3}$ & 0.855 \\
\hline$c c_{7,6}$ & 0.660 \\
\hline$c c_{8,9}$ & 0.977 \\
\hline$c c_{9,8}$ & 0.977 \\
\hline$c c_{10,13}$ & 0.765 \\
\hline$c c_{11,1}$ & 0.950 \\
\hline$c c_{12,8}$ & 0.950 \\
\hline$c c_{13,5}$ & 0.849 \\
\hline
\end{tabular}

Step 2 \& 3: Select the relevant descriptors of a given fashion theme according to the similarity degrees between theme and a selection procedure.

In Table 7, we give the ranking result of the sensory descriptors to a given fashion theme $t_{l}$. We find that $a_{1}$, $a_{11}, a_{12}$ and $a_{5}$ are the most relevant to $t_{1}$. Also, we calculate the overall correlation coefficients to remove the strongly correlated descriptors from the ranking list using eq.(4).

For example, for the descriptors $a_{1}$ and $a_{11}$, we have $R\left(a_{1}, t_{1}\right)>R\left(a_{11}, t_{1}\right)$ and $o c c_{11}=c c_{11,1}$. Then, $a_{11}$ is considered as strongly correlated with $a_{l}$, and should be removed from the list.

Using the procedure of Section 2.3, we obtain the final list of independent descriptors, ranked according to their relevancy to $t_{l}$ (see Table 8).

Table 8. List of independent descriptors relevant to $t_{l}$

\begin{tabular}{|c|c|c|c|c|c|}
\hline$a_{i}$ & $a_{1}$ & $a_{12}$ & $a_{7}$ & $a_{10}$ & $a_{3}$ \\
\hline$R\left(a_{i}, t_{l}\right)$ & 0.998 & 0.981 & 0.750 & 0.750 & 0.716 \\
$u_{i}$ & 5 & 4 & 2.5 & 2.5 & 1 \\
$\tilde{u}_{i}$ & 0.33 & 0.27 & 0.17 & 0.17 & 0.06 \\
\hline
\end{tabular}

Step 4 \& 5: Prediction of the similarity degree between a new fabric sample and a specific fashion theme.
We use the method of leave one out ${ }^{19}$ to test the effectiveness of the proposed model. Using this method, ten products are used to build the model (selection of the relevant descriptors and computation of the corresponding weights) and the remaining one is used for testing the performance of the model by calculating the RMSE value between the predicted output and the real output (Fig. 4). This procedure repeats for all combinations. Finally, the averaged RMSE value is $6.8 \%$, showing that the performance of the model is acceptable.

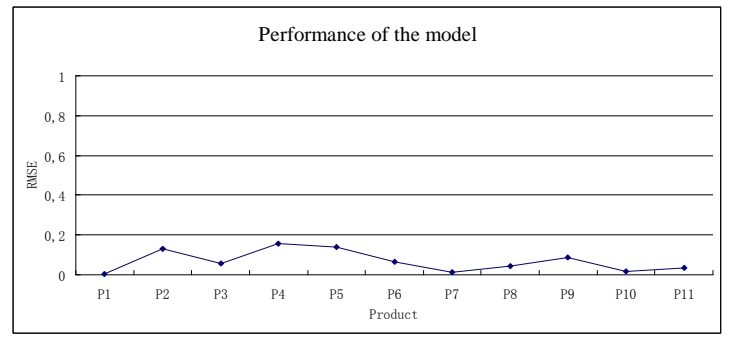

Fig. 4. The performance of the proposed model

Final, we apply the proposed model to six new fabrics $s_{12}, \ldots, s_{17}$. The similarity between these fabrics and the descriptors is evaluated by the trained panellists referring to evaluation scores of 11 existing fabrics. Then we calculate the similarity between new fabrics and the fashion theme $t_{1}$, and obtain a final ranking list of all the fabrics in Fig. 5 . We can see that fabric $s_{6}, s_{3}$, $s_{10}$, and $s_{4}$ are very close to the fashion theme $t_{1}$ while fabric $s_{14}, s_{5}, s_{9}$, and $s_{2}$ are far away from $t_{l}$. The similarity degrees of the other fabrics are between the above two groups of fabrics.

By comparing with the results obtained by the linear regression model shown in Fig. 5, the proposed method gives more reasonable results with respect to experts' professional experience on products. The proposed method can effectively treat human perception with uncertainty using fuzzy set techniques. Furthermore, the method removes the correlation effect of input variables. It is thus more efficient for industrial applications with small number of learning data. 


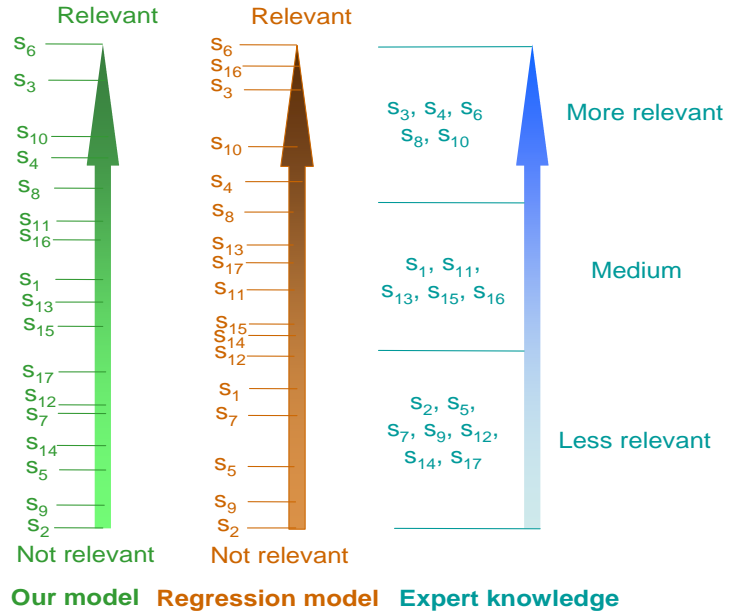

Fig. 5. Ranking list of all the fabrics

\section{Conclusions}

This paper presents an original method for predicting a fashion theme or emotional linguistic attribute from basic sensory linguistic data. This method uses both fuzzy relation and correlation computations for selecting a small set of relevant sensory descriptors and combines the relevant descriptors using a weighted aggregation operator to predict the similarity degree between new fabric and fashion themes. It helps designers to predict the consumer perception from known fabrics and design new fashion oriented products enhancing the brand image of the company. Compared with other prediction methods such as linear regression, the proposed method is more robust and interpretable owing to its capacity of treating uncertainty.

This work can also be extended to support other fashion oriented product design.

\section{References}

1. M.C.Gacula, Descriptive Sensory Analysis in Practice (Food \& Nutrition Press, Inc., Trumbull, Connecticut, 1997).

2. H. Stone, and J.L. Sidel, Sensory Evaluation Practice, (Academic Press, Inc., San Diego, CA, 2004)

3. E. Brangier and J. Barcenilla. Concevoir un produit facile à utiliser (Editions d'Organisation, Paris, 2003)

4. S. Kawabata, The development of the objective measurement of fabric handle. In Proc. of First-Japan Australia Symposium on Objective Specification of Fabric Quality, Mechanical Properties, and Performance, (Kyoto, 1982), pp. 31-59.
5. N. Pan, K.C. Yen, S.J. Zhao, S.R. Yang, A new approach to the objective evaluation of fabric handle from mechanical properties. Part I. Objective measure for total handle, Textile Research Journal, 58 (8) (1988) 438-444.

6. M. Mäkinen, H. Meinander, C. Luible, N. Magnenat-Th almann, Influence of physical parameters on fabric hand. In Proc. of the Workshop on Haptic and Tactile Perception of Deformable Objects (HAPTEX'05), (Hanover, Germany, 2005), pp. 8-16.

7. P. Volino, P. Davy, U. Bonanni, C. Luible, N. MagnenatThalmann, M. Mäkinen, \& H. Meinander, From measured physical parameters to the haptic feeling of fabric, The Visual Computer, 23(2) (2007) 133-142.

8. Y. Zhu, X. Zeng, L. Koehl, and C. Chaigneau. Development of a fuzzy selection criterion of physical features for evaluating fabric hand, In Intelligent decision making systems, (Singapore, 2009), pp. 474-479.

9. S.W. Park, Y.H. Hwang, B.C. Kang, S.W. Yeo, H.H. Cho, A fuzzy application to fabric hand evaluation. I. Application to double weft-knitted fabrics, Journal of Korean Fiber Society, 33 (10) (1996) 849-854.

10. S.W. Park, Y.H. Hwang, B.C. Kang, A fuzzy application to fabric hand evaluation. II. Application to polyester fabrics in Korea, Journal of Korean Fiber Society, 34 (2) (1997) 85-89.

11. S.W. Park, Y.H. Hwang, B.C. Kang, C.K. Bae, K. Choo, A fuzzy application to fabric hand evaluation. III. Application to warp-knitted fabrics, Journal of Korean Fiber Society, 35 (2) (1998) 119-124.

12. B. Bouchon-Meunier, R.R. Yager, L.A. Zadeh, Uncertainty in Intelligent and Information Systems, in Advances in Fuzzy Systems-Applications and Theory, (vol. 20. World Scientific, Singapore, 2000).

13. B.Pradhan, Application of an advanced fuzzy logic model for landslide susceptibility analysis, International Journal of Computational Intelligence Systems, 3 (3) (2010), 370381.

14. S. Alavandar, T. Jain and M.J. Nigam, Bacterial foraging optimized hybrid fuzzy precompensated PD control of two Link Rigid-Flexible Manipulator, International Journal of Computational Intelligence Systems, 2 (1) (2009) 51-59.

15. A. Ustundag, A hybrid model for forecasting sales in Turkish paint industry, International Journal of Computational Intelligence Systems, 2 (3) (2009) 277-287.

16. D. Dubois, and H. Prade, On the use of aggregation operations in information fusion processes, Fuzzy Sets Syst., 142 (1) (2004) 143-161.

17. B. Bouchon-Meunier, Aggregation and Fusion of Imperfect Information (Physica-Verlag, Heidelberg, 1998).

18. X. Wang, D. Ruan, and E. Kerre, Mathematics of Fuzziness - Basic Issues, (Springer - Verlag, Berlin, 2009)

19. T. Evgeniou, M. Pontil, and A. Elisseeff, Leave one out error, stability, and generalization of voting combinations of classifiers, Machine learning, 55 (1) (2004) 71-79. 